\title{
Nanometer-resolution measurement and modeling of lateral variations of the effective work function at the bilayer $\mathrm{Pt} / \mathrm{Al} / \mathrm{SiO}_{2}$ interface
}

\author{
W. Cai, K.-B. Park,* and J. P. Pelz \\ Department of Physics, The Ohio State University, Columbus, Ohio 43210, USA \\ (Received 14 August 2009; revised manuscript received 25 September 2009; published 19 October 2009)

\begin{abstract}
A ballistic electron emission microscopy (BEEM) comparison of the dependence on gate voltage of the average energy barrier of a metal bilayer $\mathrm{Pt} / \mathrm{Al} / \mathrm{SiO}_{2} / \mathrm{Si}$ sample and a $\mathrm{Pt} / \mathrm{SiO}_{2} / \mathrm{Si}$ sample suggests that the metal/oxide interface of the $\mathrm{Pt} / \mathrm{Al} / \mathrm{SiO}_{2} / \mathrm{Si}$ sample is laterally inhomogeneous at nm length scales. However, BEEM images of the bilayer sample do not show significantly larger lateral variations than observed on a (uniform) $\mathrm{Pt} / \mathrm{SiO}_{2} / \mathrm{Si}$ sample, indicating that any inhomogeneous "patches" of lower-energy barrier height have size smaller than the lateral resolution of BEEM, estimated for these samples to be $\sim 10 \mathrm{~nm}$. Finite element electrostatic simulations of an assumed inhomogeneous interface with $\mathrm{nm}$ size patches of different effective work function can fit the experimental data of the bilayer sample much better than an assumed homogenous interface, indicating that the bilayer film is laterally inhomogeneous at the nm scale.
\end{abstract}

DOI: $10.1103 /$ PhysRevB.80.165322

PACS number(s): 68.37.-d, 73.40.Qv, 73.20.At

\section{INTRODUCTION}

Future complementary metal-oxide-semiconductor (MOS) technology will require metal gates with an effective work function (EWF) that can be "tuned" to precisely adjust the transistor turn-on voltage. It was shown using macroscopic $C$ - $V$ measurements that the EWF could be adjusted using a metal bilayer as the MOS gate electrode, by adjusting the thickness of a very thin low work-function metal covered by a high work-function metal film (or vice versa) on a $\mathrm{SiO}_{2}$ film. ${ }^{1,2}$ However, it was observed that the EWF could be adjusted over a very wide thickness range of the bottom metal film (about 3-10 nm in Ref. 2). This is surprising because one would naively expect that the effects of the top metal film would be effectively screened once the bottom metal film was more than a few monolayers thick. However, as discussed by Jeon et al., ${ }^{2}$ this behavior would make more sense if the metal/ $\mathrm{SiO}_{2}$ interface were actually laterally inhomogeneous, with small "pinholes" in the bottom film filled in by the top film. The tunability would then come from the decrease in average pinhole coverage and diameter with increasing bottom metal thickness. If so, it may be possible to use the nm-resolution ballistic electron emission microscopy (BEEM) technique ${ }^{3,4}$ to reveal this inhomogeneity.

Here we report BEEM experiments performed on $5 \mathrm{~nm}-\mathrm{Pt} / 10 \mathrm{~nm}-\mathrm{SiO}_{2} / \mathrm{Si}$ and $5 \mathrm{~nm}-\mathrm{Pt} / 1.4 \mathrm{~nm}-\mathrm{Al} / \mathrm{SiO}_{2} / \mathrm{Si}$ gate stack MOS structures. Direct BEEM images do not reveal significantly larger spatial variations on the metal bilayer sample than observed on the uniform Pt film sample, indicating that any inhomogeneities along the metal bilayer $/ \mathrm{SiO}_{2}$ sample are smaller than the estimated $\sim 10 \mathrm{~nm}$ BEEM spatial resolution for a sample with a $\sim 5$-nm-thick Pt film. However, BEEM measurements of the dependence on the gate voltage of the average energy barrier height show a different behavior for a metal bilayer $/ \mathrm{SiO}_{2}$ sample as compared with the uniform $\mathrm{Pt} / \mathrm{SiO}_{2} / \mathrm{Si}$ sample, consistent with finite element electrostatic simulations that assume nm-scale "patches" of different EWF for the metal bilayer sample, indicating that the bilayer sample is in fact inhomogeneous at the nm scale.

\section{EXPERIMENT}

\section{A. BEEM}

BEEM, which is an extension of scanning tunneling microscopy (STM), was first used by Kaiser and Bell to study the microscopic properties of metal/semiconductor Schottky barriers $^{3,4}$ and was subsequently applied to the study of MOS structures. ${ }^{5-8}$ The configuration of the BEEM technique for MOS structures is shown in Fig. 1(a). When the tip is brought close to the metal film with a negative bias [corresponding to $V_{T}>0$ with polarities defined in Fig. 1(a)], electrons will be locally injected from the tip into the metal film with a range of energy close to the Fermi level of tip, which is $e V_{T}$ above the Fermi level in the metal film. The hotelectron energy, location, and flux can be controlled by varying the voltage, position, and tunnel current of the tip, respectively. Provided the metal film is sufficiently thin, a fraction of the injected hot electrons can transport across the metal film and reach the metal/oxide interface without losing significant energy. Some of the hot electrons having sufficient energy to overcome the maximum energy barrier in the oxide can transport across the oxide to reach the Si substrate and be collected as the external current $I_{c}$, while others will be scattered back into the metal or trapped in the oxide. The local energy barrier height $\phi_{\text {bar }}$ in the oxide film can be determined as $\phi_{\text {bar }}=e V_{\text {th }}$, where $V_{\text {th }}$ is the threshold tip voltage for electrons to cross the oxide film. Figures 1(b) and 1(c) also show that the energy barrier in the oxide can be changed by changing the gate bias $V_{\text {gate }}$ defined here as the voltage of the metal film relative to the Si substrate. For the "forward" electric field case as in Fig. 1(b), BEEM probes the energy barrier at the metal/oxide interface. The energy barrier will decrease slightly because of "image-force lowering"9 as $V_{\text {gate }}$ is made more negative. For the "reverse" electric field case, as in Fig. 1(c), the maximum energy barrier location switches to the oxide/Si interface, and the energy barrier will increase linearly with $V_{\text {gate }}$. We note that Fig. 1 assumes no trapped charge in the oxide film ${ }^{7,8,10}$ and a laterally homogeneous interfaces. The effect of trapped charge and inhomogeneity on the measured barrier height will be discussed later in this paper. 
(a)

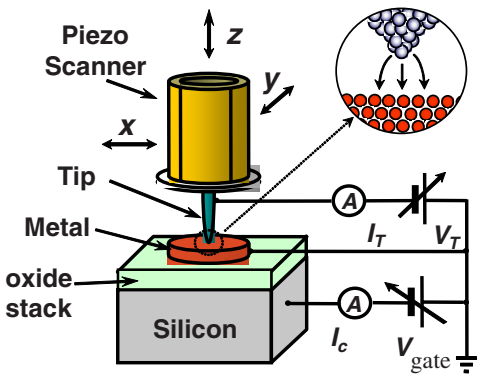

(b)
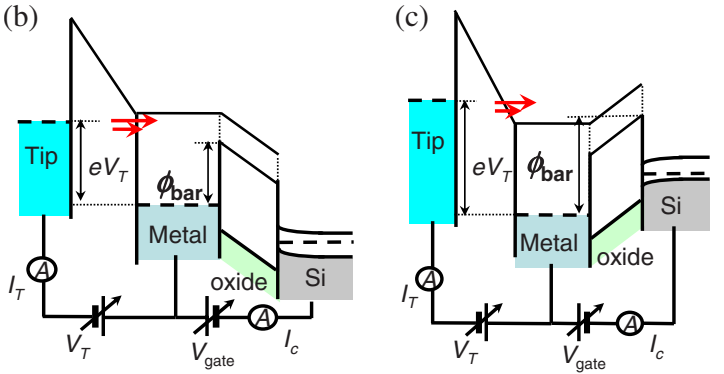

FIG. 1. (Color online) Schematic configuration of BEEM experiment on MOS structures (a) and the corresponding energy-level diagrams for both forward (b) and reverse (c) oxide electric field. The horizontal axis in (b) and (c) corresponds to the $z$ axis in (a). The metal/oxide (oxide/Si) interface is probed in (b) [(c)]. In (c), hot electrons from the metal side must cross the oxide film against an electric field to contribute to the substrate current $I_{c}$.

\section{B. Sample preparation}

$\mathrm{Si}(100)$ wafers (n type with resistivity 0.004 $-0.02 \Omega \mathrm{cm}$ ) with $10 \mathrm{~nm}$ of high quality commercial oxide were cut into $9 \times 20 \mathrm{~mm}$ pieces, degreased using a sequence of ultrasonic trichloroethylene, acetone, and methanol, and finally rinsed in de-ionized water. Samples were then immediately introduced into an ultrahigh vacuum (UHV) preparation chamber and attached UHV STM/BEEM system ${ }^{7}$ and heated to a pyrometer reading of $\sim 340{ }^{\circ} \mathrm{C}$ for $10 \mathrm{~min}$ to desorb water and hydrocarbons. Then either a $\sim 5$-nm-thick $\mathrm{Pt}$ or a 5-nm-Pt/1.4-nm-Al metal bilayer film was deposited by electron-beam evaporation through a shadow mask to form a number of $0.5 \mathrm{~mm}^{2}$ diameter dots. Samples were then transported in situ to the attached STM/BEEM chamber without any postmetallization anneal. A 0.1-mm-diameter Au wire positioned with a mechanical manipulator was used to contact particular metal dots for the BEEM measurements.

\section{RESULTS AND DISCUSSION}

\section{A. Measurements}

Figure 2(a) shows example average BEEM $I_{c}-V_{T}$ curves for the $5-\mathrm{nm}-\mathrm{Pt} / \mathrm{SiO}_{2} / \mathrm{Si}$ (open squares) and 5-nm-Pt/1.4-nm-Al/ $\mathrm{SiO}_{2} / \mathrm{Si}$ (open circles) samples. Each curve represents an average of $\sim 45$ individual BEEM $I_{c}-V_{T}$ curves taken sequentially at different locations. The BellKaiser model ${ }^{4}$ was used to fit (solid lines) the experimental BEEM curve to determine the threshold voltage $V_{\text {th }}$ and, in turn, the energy barrier $\phi_{\mathrm{bar}}=e V_{\mathrm{th}}$, using a $0.5 \mathrm{~V}$ range of data centered self-consistently around the best-fit value of $V_{\text {th. }}$. As can be seen in Fig. 2(a), at $V_{\text {gate }}=0 \mathrm{~V}, \phi_{\text {bar }}$ for the
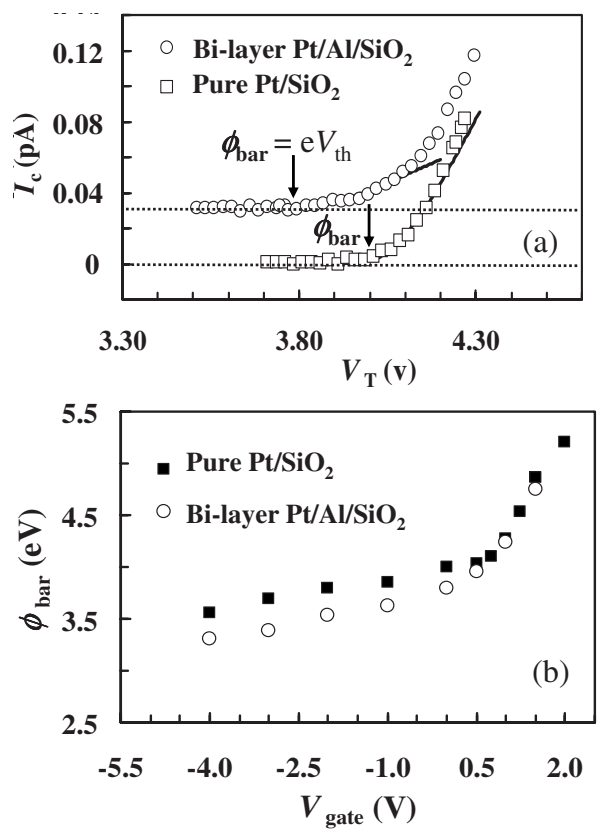

FIG. 2. (a) Typical BEEM $I_{c}-V_{T}$ data for bilayer $\mathrm{Pt} / \mathrm{Al} / \mathrm{SiO}_{2}$ (open circle) and pure $\mathrm{Pt} / \mathrm{SiO}_{2}$ samples (open square) and BellKaiser model fits (solid lines) and best-fit barrier heights (arrows) at $V_{\text {gate }}=0 \mathrm{~V}$. Each data is an average of $\sim 45$ individual $I_{c}-V_{T}$ curves measured at different locations. Top curve has been vertically offset for clarity. Tip current was $10 \mathrm{nA}$. (b) Best-fit barrier heights vs $V_{\text {gate }}$ determined by BEEM for both pure $\mathrm{Pt} / \mathrm{SiO}_{2}$ (solid square) and bilayer $\mathrm{Pt} / \mathrm{Al} / \mathrm{SiO}_{2}$ (open circle).

metal bilayer sample is $\sim 0.2 \mathrm{eV}$ lower than for the pure $\mathrm{Pt} / \mathrm{SiO}_{2}$ sample, which shows that by inserting a lower work-function metal film the effective work function of the gate stack is indeed adjustable (effective work function is used instead of work function since the band alignment at the metal/oxide interface can depend on other factors besides the metal work function such as material-dependent charge transfer at the metal/oxide interface, etc.), in agreement with prior studies. ${ }^{1,2}$

Figure 2(b) plots the measured barrier height $\phi_{\text {bar }}$ [as determined from BEEM $I_{c}-V_{T}$ curves such as shown in Fig. $2(\mathrm{a})]$ as a function of $V_{\text {gate }}$. One can see several important features in this data: (a) $\phi_{\text {bar }}$ is lower for the bilayer sample (open circle) than the pure-Pt sample (solid square) below $\sim 0.5 \mathrm{~V}$ but becomes essentially the same at higher $V_{\text {gate }}$ (b) Both curves have a transition "knee" to a larger slope close to $\sim 0.5 \mathrm{~V}$. (c) The transition is more abrupt for the pure-Pt film than the bilayer film. As discussed above, for negative $V_{\text {gate }}$ [which corresponds to the forward electric field as in Fig. 1(b)] BEEM probes the energy barrier at the metal/ $\mathrm{SiO}_{2}$ interface, so any difference in the EWF between pure-Pt and the Pt/Al bilayer should be directly observable as a difference in the energy barrier $\phi_{\text {bar }}$ measured by BEEM. The weak $\phi_{\text {bar }}$ dependence on $V_{\text {gate }}$ for both samples in this forward electric field case is consistent with the image-force lowering effect ${ }^{9}$ expected at the metal $/ \mathrm{SiO}_{2}$ interface. For large positive $V_{\text {gate }}$ [which corresponds to the reverse electric field as in Fig. 1(c)], the maximum energy barrier location switches to the $\mathrm{SiO}_{2} / \mathrm{Si}$ interface, with a subsequent strong 

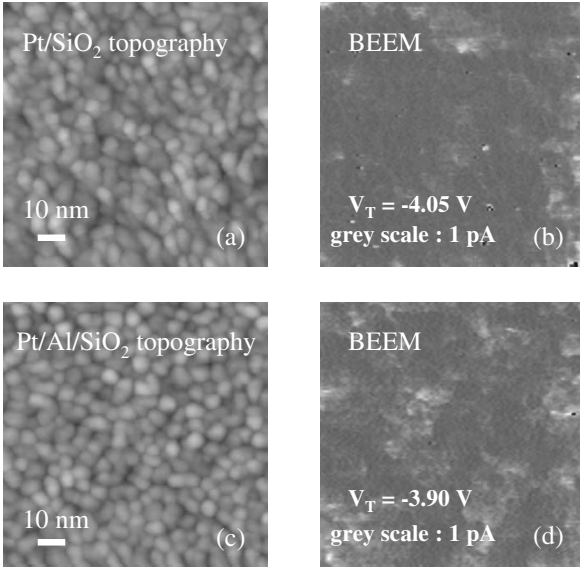

FIG. 3. STM topography of $\mathrm{Pt} / \mathrm{SiO}_{2}$ (a) and $\mathrm{Pt} / \mathrm{Al} / \mathrm{SiO}_{2}$ (c) and corresponding BEEM images [(b) and (d)] with $V_{\text {gate }}=-1$ V. Tip current was $10 \mathrm{nA}$. The STM topography and BEEM image are taken simultaneously.

direct dependence of the barrier height $\phi_{\text {bar }}$ on $V_{\text {gate }}$. In this case, the barrier height $\phi_{\text {bar }}$ is mainly determined by the electron affinity difference between $\mathrm{SiO}_{2}$ and $\mathrm{Si}$, the doping of the $\mathrm{Si}$, and the value of $V_{\text {gate }}$, but is only weakly dependent on the metal EWF. Consequently, $\phi_{\text {bar }}$ for both the pure-Pt and bilayer samples should become nearly the same at large positive $V_{\text {gate }}$, as observed in Fig. 2(b). The transition knee close to $\sim 0.5 \mathrm{~V}$ for both samples corresponds to the value of $V_{\text {gate }}$ at which the average electric field in oxide $E_{o x}$ is zero. We note that zero $E_{o x}$ typically does not occur at zero $V_{\text {gate }}$ due to the contact potential difference between the metal and the semiconductor substrate.

We next consider whether BEEM is able to directly observe increased spatial inhomogeneity at the metal bilayer $/ \mathrm{SiO}_{2}$ interface as compared to the pure $\mathrm{Pt} / \mathrm{SiO}_{2}$ interface. Typical BEEM images at $V_{\text {gate }}=-1 \mathrm{~V}$ (which corresponds to the forward electric field case for both samples) and $I_{T}=10 \mathrm{nA}$ on the $5-\mathrm{nm}-\mathrm{Pt} / \mathrm{SiO}_{2} / \mathrm{Si}$ and 5-nm-Pt/1.4-nm-Al/ $\mathrm{SiO}_{2} / \mathrm{Si}$ samples are shown in Fig. 3. The forward electric field condition is critical for BEEM to directly observe any spatial inhomogeneity in the energy barrier at metal/oxide interface since BEEM probes the energy barrier at front metal/oxide interface only for forward electric field. The tip voltages in Fig. 3(b) and 3(d) were chosen to be slightly above the respective threshold voltages for the two samples at $V_{\text {gate }}=-1 \mathrm{~V}$ in order to reduce the amount of charge injected into the $\mathrm{SiO}_{2}$ film and so reduce possible effects of trapped charge on the BEEM images. ${ }^{7}$ In Fig. 3, both the STM topography [(a) and (c)] and BEEM images $[(b)$ and (d)] of pure-Pt and metal bilayer samples look very similar. The BEEM images of both samples show inhomogeneous patches of larger and smaller BEEM currents. Such variations in the local BEEM current could result from local variations in $\phi_{\mathrm{bar}}$ but could also result from local variations in electron transmittance through the metal film (due to variations in metal thickness or the crystal orientation of grains in the polycrystalline metal film) or across the metal/ oxide interface.

In order to determine whether the bilayer sample has larger variations in local barrier height than the pure-Pt sample, we analyzed about 45 individual BEEM $I_{c}-V_{T}$ curves measured at different locations across each of the two samples. We found that the standard deviation (SD) of $\phi_{\text {bar }}$ was about the same for both the pure $\mathrm{Pt} / \mathrm{SiO}_{2}$ (SD $\cong 85 \mathrm{meV})$ and the metal bilayer $/ \mathrm{SiO}_{2}(\mathrm{SD} \cong 95 \mathrm{meV})$ samples. This indicates that either the two samples have roughly the same degree of inhomogeneity in local barrier height, or that the spatial resolution of BEEM is not sufficient to directly resolve large local variations in barrier height, or that the measurement uncertainty for a single BEEM $I_{c}-V_{T}$ curve is larger than the local variations in barrier height. We estimate the BEEM spatial resolution for these samples to be $\sim 8-10 \mathrm{~nm}$ based on previous BEEM studies $^{11,12}$ of 4-nm-thick, 5-nm-thick, and 8-nm-thick Pt films deposited on $4 \mathrm{H}-\mathrm{SiC}$ samples with embedded stacking faults that behaved as quantum wells of very narrow width $(0.5-1.25 \mathrm{~nm})$, where the Schottky barrier height $(\mathrm{SBH})$ over the stacking fault was much smaller than over the surrounding $\mathrm{Pt} / \mathrm{SiC}$. In those studies, the averaged full width at half maximum of BEEM profiles over the stacking faults was found to range from $\sim 8 \mathrm{~nm}$ for the 4-nm-thick Pt film to $\sim 10 \mathrm{~nm}$ for the 8-nm-thick Pt film, most likely due to lateral spreading of the injected hot electrons in the Pt film before entering the semiconductor. ${ }^{13}$ So if the metal bilayer sample has very small $(<\sim 10 \mathrm{~nm})$ local patches with significantly varying barrier height, they may not be directly visible in BEEM images or by measurement of local BEEM $I_{c}-V_{T}$ curves. We note that if inhomogeneity in the bilayer film is caused by pinholes in 1.4-nm-thick bottom Al film, the expected length scale would be comparable to the average grain size of the $\mathrm{Al}$ film, which should have, as an upper limit, the $5-10 \mathrm{~nm}$ grain size of the complete bilayer film determined from Fig. 3(c). However, very small patches of varying local barrier height should still produce observable effects in the spatially averaged barrier height, as discussed below.

\section{B. Modeling the dependence of the spatially averaged barrier height $\phi_{\text {bar }}$ on $V_{\text {gate }}$}

We next examine whether we can theoretically fit the experimental measurements of the spatially averaged barrier height $\phi_{\text {bar }}$ vs $V_{\text {gate }}$ for pure-Pt and metal bilayer samples that are shown in Fig. 2(b). Assuming a uniform interface and no trapped charge in the oxide, the dependence of the energy barrier $\phi_{\text {bar }}$ (including image-force lowering effects) on $V_{\text {gate }}$ can be calculated based on ${ }^{9}$ the Si electron affinity $\chi_{\mathrm{Si}}$, the $\mathrm{Si}$ doping $N_{\mathrm{d}}$, the $\mathrm{SiO}_{2}$ electron affinity $\chi_{\mathrm{SiO}_{2}}$, the $\mathrm{SiO}_{2}$ layer thickness $t_{\text {oxide, }}$, the $\mathrm{SiO}_{2}$ high-frequency dielectric constant $\varepsilon_{\text {high }}$, and the work function of metal. The solid lines in Fig. 4(a) and 4(b) are the theoretical calculated results of $\phi_{\text {bar }}$ vs $V_{\text {gate }}$ for pure-Pt and metal bilayer samples, respectively. Known parameters used in the calculation are $\chi_{\mathrm{Si}}=4.05 \mathrm{eV}$, $N_{\mathrm{d}}=5 \times 10^{18} / \mathrm{cm}^{3}, \chi_{\mathrm{SiO}_{2}}=0.89 \mathrm{eV}, t_{\text {oxide }}=10 \mathrm{~nm}$, and $\varepsilon_{\text {high }}$ $=2.15$. The effective work function $W$ of the metal used in the calculation is adjustable to fit the experimental data of $\phi_{\text {bar }}$ vs $V_{\text {gate }}$ for both samples. Figure 4(a) shows that the theoretical calculation fits the experimental data of pure-Pt sample very well with the best fit of $W_{\mathrm{Pt}}=5.1 \mathrm{eV}$, which in 

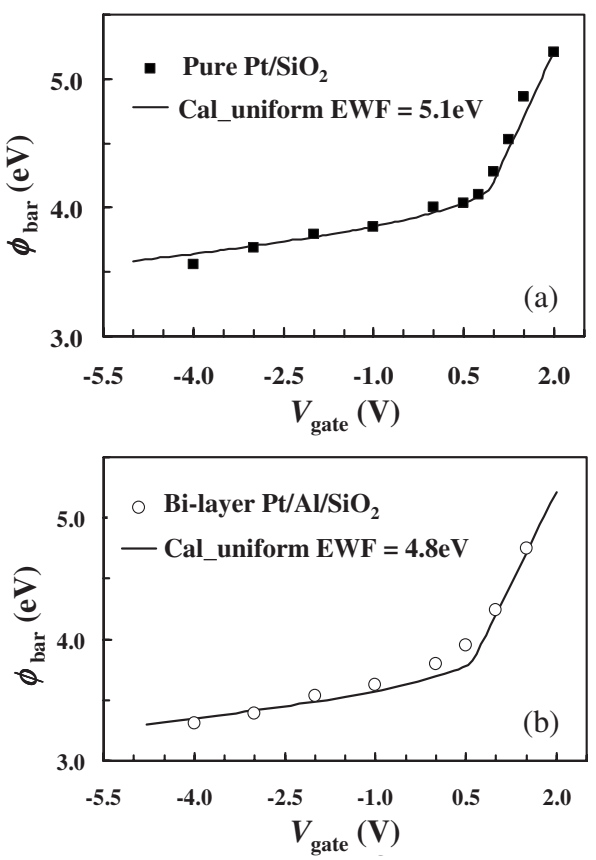

FIG. 4. BEEM energy barrier $\phi_{\text {bar }}$ dependence on $V_{\text {gate }}$ data (symbols) and model fits (solid lines) by assuming a uniform interface for both pure $\mathrm{Pt} / \mathrm{SiO}_{2}$ (a) and bilayer $\mathrm{Pt} / \mathrm{Al} / \mathrm{SiO}_{2}$. In (b), there is a small curvature of BEEM data around the flat band voltage that cannot be fitted by assuming a uniform interface.

turn suggests that the pure-Pt sample interface is uniform and does not have significant oxide trapped charge (see below). However, the best fit (with $W_{\mathrm{Pt} / \mathrm{Al}}=4.8 \mathrm{eV}$ ) is much worse for the metal bilayer sample, as shown in Fig. 4(b). In particular, the metal bilayer sample has a much more gradual change in $\phi_{\text {bar }}$ vs $V_{\text {gate }}$ close to the knee at $\sim 0.5 \mathrm{~V}$ than the pure-Pt sample.

We next consider two possible origins of the gradual change in $\phi_{\text {bar }}$ vs $V_{\text {gate }}$ at $V_{\text {gate }} \cong 0.5 \mathrm{~V}$ for the bilayer sample. (1) It may come from a large trapped charge density in the $\mathrm{SiO}_{2}$, which was previously shown to produce significant "rounding" of $\phi_{\text {bar }}$ vs $V_{\text {gate }}$ curves. ${ }^{10}$ (2) It may be due to an inhomogeneous EWF at the metal/oxide interface. For the first possible origin, by assuming a uniform EWF at the metal/oxide interface but with trapped charge in the oxide, we can indeed fit the experimental data of $\phi_{\text {bar }}$ vs $V_{\text {gate }}$ of bilayer sample pretty well (not shown) by assuming $W_{\mathrm{Pt} / \mathrm{Al}}$ $=4.7 \mathrm{eV}$ and a (large) uniform trapped charge density in the $\mathrm{SiO}_{2}$ of $n_{\text {bulk }}=-3.6 \times 10^{18} \mathrm{~cm}^{-3}$ (which corresponds to $-3.6 \times 10^{12} \mathrm{~cm}^{-2}$ projected areal charge density for a $10-\mathrm{nm}-$ thick $\mathrm{SiO}_{2}$ ). However, such a large trapped charge density would also result in $\sim+1 \mathrm{~V}$ flat band voltage shift in $C-V$ measurements ${ }^{9}$ of the bilayer sample, as compared to the pure-Pt sample, which was not observed. We conclude that the more gradual change in the $\phi_{\text {bar }}$ vs $V_{\text {gate }}$ behavior of the bilayer sample is unlikely to be simply due to a large trapped charge density in the oxide.

Tung ${ }^{14,15}$ considered the expected electrical behavior of metal/semiconductor contacts with a spatially inhomogeneous barrier height $\phi_{\text {bar }}(x, y)$, where the lateral length scale of the inhomogeneity is comparable to or smaller than the depletion width of the semiconductor. He showed that the
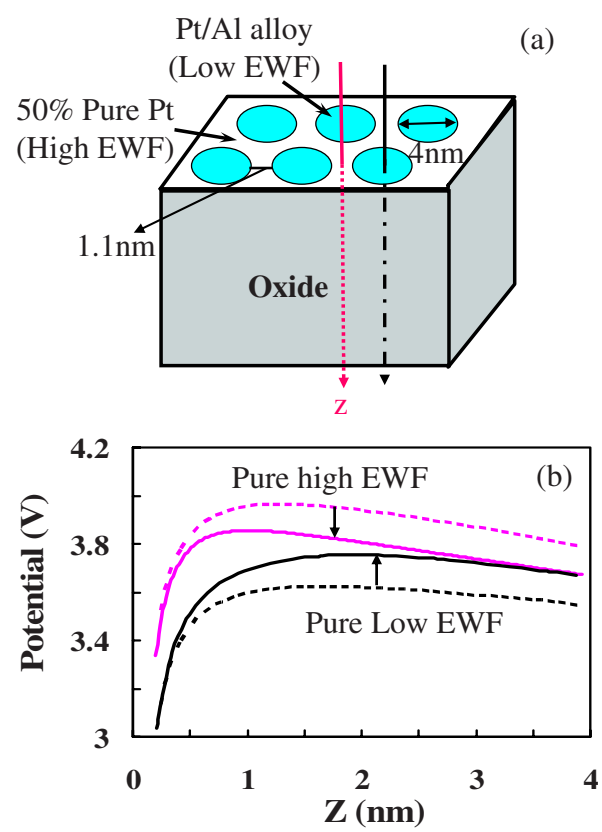

FIG. 5. (Color online) (a) The assumed inhomogeneous geometry at the bilayer $\mathrm{Pt} / \mathrm{Al} / \mathrm{SiO}_{2}$ interface. $50 \% \mathrm{Pt} / \mathrm{Al}$ alloy circular patches with $4 \mathrm{~nm}$ diameter are uniformly distributed and surrounded by pure-Pt patches at the metal/oxide interface. (b) The demonstration of Tung effect. The potential under a uniform high EWF patch (upper dashed line) will be pulled down (upper solid line) and the potential under a uniform low EWF patch (lower dashed line) will be pulled up (lower solid line) by considering the inhomogeneous geometry as shown in (a).

electrostatic potential below low-barrier height regions could experience a "potential pinch-off" effect produced by surrounding high-barrier height regions that would strongly increase the local barrier height. For the metal- $\mathrm{SiO}_{2}-\mathrm{Si}$ structures considered here, such potential pinch-off effects should become strong when the size of low-barrier patches is less than the thickness of the $\mathrm{SiO}_{2}$ film (10 nm in our case), as illustrated in Fig. 5. The circular patches in Fig. 5(a) represent low $\phi_{\text {bar }}(x, y)$ regions at the metal-oxide interface (produced by low EWF Pt/Al alloy regions) that are surrounded by high $\phi_{\text {bar }}(x, y)$ regions (produced by high EWF pure-Pt regions). The upper dashed line and lower dashed line [Fig. $5(\mathrm{~b})]$ are the calculated vertical potential profiles for a uniform interface (with no trapped oxide charge), using the known parameters listed above and assuming an EWF of 5.1 $\mathrm{eV}$ and $4.7 \mathrm{eV}$, respectively. As expected, the metal-oxide barrier heights (determined by the maximum in the potential profile) for these two cases differ by $\sim 0.4 \mathrm{eV}$, which is the difference of the assumed EWFs of the high- and low-barrier regions. For comparison, the upper and lower solid lines in Fig. 5(b) show calculated potential profiles along two different paths normal to an inhomogeneous interface, consisting of a $50 \%$ coverage of 4-nm-diameter circular patches of a low $(4.7 \mathrm{eV})$ EWF metal surrounded by a high (5.1 eV) EWF metal, determined by finite element electrostatic simulations done with the commercial software package FLEX PDE (Ref. 16) (image-force lowering was added after calculation). For the finite element electrostatic simulations, Dirichlet boundary conditions were assumed at the metal/ $\mathrm{SiO}_{2}$ and $\mathrm{SiO}_{2} / \mathrm{Si}$ 


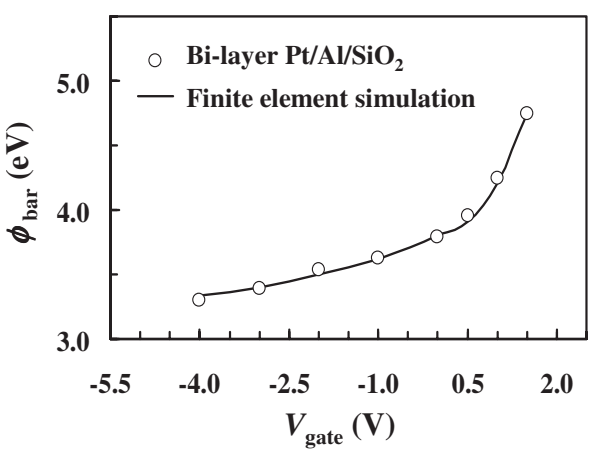

FIG. 6. BEEM energy barrier $\phi_{\text {bar }}$ dependence on $V_{\text {gate }}$ data (open circle) and finite element simulation fit (solid line) of bilayer $\mathrm{Pt} / \mathrm{Al} / \mathrm{SiO}_{2}$ by assuming inhomogeneous metal/oxide interface with certain geometry as shown in Fig. 5(a).

interfaces with the values of the electron potential energy given by $\mathrm{W}_{\text {metal }}-\chi_{\mathrm{SiO}_{2}}$ and by $\left(\chi_{\mathrm{Si}^{-}}-\chi_{\mathrm{SiO}_{2}}\right)+q V_{\text {gate }}$, respectively. Heavy $n$ doping of the Si is assumed. Periodic boundary conditions were assumed parallel to the interface. The lower and upper solid lines in Fig. 5(b) show potential profiles along a path through the center of a low EWF patch and midway between the patches, respectively. We see from these two curves that the local barrier height (the maximum along the potential profile) through a low-EWF region is pulled $u p$ by the surrounding high-EWF regions, (as compared to a uniform low-EWF interface) while that barrier through a high-EWF region is pulled down by the nearby low-EWF regions. The barrier height through other locations on this surface should fall between these two extreme values.

In order to compare these simulations of an inhomogeneous interface with the measured BEEM data shown in Fig. 4 , we used the following procedure. For a given value of $V_{\text {gate }}$, the maximum and minimum barrier heights were determined as described above and then for each barrier an expected BEEM $I_{c^{-}}-V_{T}$ curve was simulated (using the BellKaiser model ${ }^{4}$ ), and the two simulated BEEM $I_{c}-V_{T}$ curves were averaged together and then were fit using the BellKaiser model to determine an "average" barrier height for that value of $V_{\text {gate }}$. This procedure was then repeated for other values of $V_{\text {gate }}$, producing the simulated $\phi_{\text {bar }}$ vs $V_{\text {gate }}$ curve shown in Fig. 6. We see that this simulated curve for an inhomogeneous interface fits the measured data for the bilayer sample much better than does a model that assumes a uniform interface [shown in Fig. 4(b)]. We note that since lateral potential variations produced by the inhomogeneous metal $/ \mathrm{SiO}_{2}$ interface could deflect electrons preferentially toward the low-barrier regions located below the low effective work-function patches, we also considered the extreme case, where $100 \%$ of the BEEM electrons could find the low-barrier regions. This resulted in almost the same simulated $\phi_{\text {bar }}$ vs $V_{\text {gate }}$ curve shown in Fig. 6. Since threshold values in BEEM $I_{c}-V_{T}$ curve fits are mostly determined by the lowest threshold present, it is to be expected that fits to the "50\%-low and 50\%-high" and the "100\%-low" simulated curves should produce similar threshold values. We note that other combinations of small patch size $(<10 \mathrm{~nm})$, coverage, and assumed values for the low- and high-EWF regions can also produce good fits to the bilayer data; but a model that assumes a uniform interface cannot. Furthermore, our simulations indicate that a model with a larger patch size produces progressively worse fits as the patch size is increased above $\sim 10 \mathrm{~nm}$ (the $\mathrm{SiO}_{2}$ film thickness) because the potential pinch-off effects of the bilayer sample become progressively weaker. For a patch size larger than $20 \mathrm{~nm}$, the simulation essentially produces a "parallel conduction model" with little potential pinch-off effect. The best fit in this case (with EWF of 4.75 and $5.10 \mathrm{eV}$ for the two regions and 50\% coverage for the low EWF patches) is very close to the solid line fit in Fig. 4(b), which assumed a uniform EWF of 4.8 $\mathrm{eV}$. This is because the low-EWF patches essentially determine the threshold voltage of the simulated BEEM $I_{c}-V_{T}$ curves and exhibit almost the same $\phi_{\text {bar }}$ vs $V_{\text {gate }}$ behavior as a uniform interface. The simulations can only reproduce the more gradual $\phi_{\text {bar }}$ vs $V_{\text {gate }}$ behavior measured for the bilayer sample if a smaller $(<\sim 10 \mathrm{~nm})$ patch size is assumed.

\section{CONCLUSIONS}

In summary, a comparison of BEEM measurements made on pure $\mathrm{Pt} / \mathrm{SiO}_{2} / \mathrm{Si}$ and bilayer $\mathrm{Pt} / \mathrm{Al} / \mathrm{SiO}_{2} / \mathrm{Si}$ samples reveal a qualitatively different dependence of the measured barrier height on gate voltage, suggesting that the metal/ oxide interface of bilayer $\mathrm{Pt} / \mathrm{Al} / \mathrm{SiO}_{2} / \mathrm{Si}$ sample is laterally inhomogeneous at the $\mathrm{nm}$ scale. This proposal is supported by finite element electrostatic simulations, which show that a model with a uniform interface can adequately describe the data for the pure-Pt sample, but that a model interface with small $(<10 \mathrm{~nm})$ patches of different EWF is necessary to describe the data for the bilayer sample. We could not directly see these small patches in BEEM images, most likely because they are smaller than the estimated $\sim 10 \mathrm{~nm}$ BEEM spatial resolution for these samples. This study suggests that the large reported "tunability" of the EWF of metal bilayer samples ${ }^{1,2}$ is due to an inhomogeneous metal/ $\mathrm{SiO}_{2}$ interface, with the top metal filling in pinholes in the bottom metal film.

\section{ACKNOWLEDGMENT}

This work was supported by the National Science Foundation Grant No. DMR-0505165. 
*Present address: School of Electrical and Computer Engineering, Ulsan National Institute of Science and Technology, Ulsan, Korea.

${ }^{1}$ W. Gao, J. F. Conley, and Y. Ono, Mater. Res. Soc. Symp. Proc. 765, D1.4.1 (2003).

${ }^{2}$ I. S. Jeon, J. Lee, P. Zhao, P. Sivasubramani, T. Oh, H. J. Kim, D. Cha, J. Huang, M. J. Kim, B. E. Gnade, J. Kim, and R. M. Wallace, Tech. Dig. - Int. Electron Devices Meet. 2004, 303.

${ }^{3}$ W. J. Kaiser and L. D. Bell, Phys. Rev. Lett. 60, 1406 (1988).

${ }^{4}$ L. D. Bell and W. J. Kaiser, Phys. Rev. Lett. 61, 2368 (1988).

${ }^{5}$ R. Ludeke, A. Bauer, and E. Cartier, Appl. Phys. Lett. 66, 730 (1995).

${ }^{6}$ R. Ludeke, A. Bauer, and E. Cartier, J. Vac. Sci. Technol. B 13, 1830 (1995).

${ }^{7}$ B. Kaczer, Z. Meng, and J. P. Pelz, Phys. Rev. Lett. 77, 91 (1996).
${ }^{8}$ B. Kaczer and J. P. Pelz, J. Vac. Sci. Technol. B 14, 2864 (1996).

${ }^{9}$ S. M. Sze, Physics of Semiconductor Devices (Wiley, New York, 1981).

${ }^{10}$ B. Kaczer, H.-J. Im, J. P. Pelz, and R. M. Wallace, Appl. Phys. Lett. 73, 1871 (1998).

${ }^{11}$ Y. Ding, K.-B. Park, J. P. Pelz, K. C. Palle, M. K. Mikhov, B. J. Skromme, H. Meidia, and S. Mahajan, Phys. Rev. B 69, 041305(R) (2004).

${ }^{12}$ K.-B. Park, J. P. Pelz, J. Grim, and M. Skowronski, Appl. Phys. Lett. 87, 232103 (2005).

${ }^{13}$ C. Tivarus, J. P. Pelz, M. K. Hudait, and S. A. Ringel, Appl. Phys. Lett. 87, 182105 (2005).

${ }^{14}$ R. T. Tung, Appl. Phys. Lett. 58, 2821 (1991).

${ }^{15}$ R. T. Tung, Phys. Rev. B 45, 13509 (1992).

${ }^{16} \mathrm{PDE}$ Solutions Inc. (Antioch, CA). 